\title{
Role of Radiotherapy-Induced Malignancies in Patients with Both Breast and Lung Cancer Diagnoses
}

\author{
Eric Khang Nguyen"1, Garth Andrew Nicholas², Xinni Song2 ${ }^{2 *}$ \\ ${ }^{1}$ Department of Radiation Oncology, Juravinski Cancer Centre, Hamilton, Canada \\ ${ }^{2}$ Department of Internal Medicine, Division of Medical Oncology, The Ottawa Hospital Regional \\ Cancer Centre, Ottawa, Canada \\ Email: xsong@toh.ca
}

How to cite this paper: Nguyen, E.K. Nicholas, G.A. and Song, X.N. (2018) Role of Radiotherapy-Induced Malignancies in Patients with Both Breast and Lung Cancer Diagnoses. Advances in Breast Cancer Research, 7, 231-242.

https://doi.org/10.4236/abcr.2018.73014

Received: June 8, 2018

Accepted: July 16, 2018

Published: July 19, 2018

Copyright (C) 2018 by authors and Scientific Research Publishing Inc. This work is licensed under the Creative Commons Attribution International License (CC BY 4.0).

http://creativecommons.org/licenses/by/4.0/

\begin{abstract}
Background: Breast and lung cancer are two of the most commonly diagnosed cancers in North America. While patients are living longer with advances in treatment and supportive care, some patients are being diagnosed with a second malignancy. The primary objective in this study was to assess the correlation between the development of an ipsilateral lung cancer or breast cancer, and prior radiation therapy. In addition, we sought to report the survival outcomes of patients in these clinical scenarios. Methods: We conducted a single institution (the Ottawa Hospital Cancer Centre) retrospective review of patients with the diagnoses of both breast and lung cancer treated between 1995 and 2013. Patients were included if they received radiation for a breast primary, and subsequently developed an ipsilateral lung primary, or vice-versa. Data included patient demographics, lifestyle factors, tumor location and subtype, cancer stages, treatment modalities, and survival outcomes. Results: Of 252 patients included in the study, 217 patients developed a breast primary first, with 35 patients developing a lung primary first. Median disease-free survival from the second primary diagnosis was 36 months in breast primary first patients, and 59 months in the lung primary first cohort. There was no significant correlation between the laterality of radiation treatment and side of second primary based on Fisher's exact test. Conclusions: Our data reveal no association between side of radiation treatment and subsequent cancer development. The benefits of radiotherapy outweigh the risk of radiation-induced primaries. Longer term studies with matched patient cohorts are required to further assess treatment and lifestyle factors that may contribute towards the development of second malignancies.
\end{abstract}




\section{Keywords}

Breast, Lung, Cancer, Radiotherapy-Induced, Ipsilateral

\section{Introduction}

Lung cancer and breast cancer are two of the most commonly diagnosed malignancies in North America and rank first and second among cancer-related deaths respectively. Given the high incidence of these cancers, some patients may have the risk of developing multiple primaries in their lifetime.

There have been several studies that reported an increased risk of second primary cancers in patients with an initial diagnosis of breast cancer [1]-[6]. The increased propensity to develop a second primary malignancy has been thought to be related to common genetic, hormonal, or environmental risk factors. For example, BRCA-1 and BRCA-2 genes are known to be involved in inherited susceptibility to breast and ovarian cancer [7]. Smoking is the most significant risk factor associated with the development of primary lung cancer. Following this, prior exposure to ionizing radiation has been shown to play a role in the development of new lung primaries with reported evidence in atomic bomb survivors, uranium miners, and medically irradiated cancer populations [8] [9] [10]. There is less literature evaluating the development of secondary breast cancers following an initial diagnosis of lung cancer.

Historically, individuals with cancer have rarely survived past the latency periods inherent to the development of treatment-induced malignancies, but improvements in systemic and radiation therapy have increased the time for these second primaries to emerge [11]. In the past 15 years, advances in cancer diagnostics, therapy and supportive care have significantly increased disease-free survival and overall survival rates in cancer patients. Effective cancer treatment has resulted in many cancer patients living 10 or more years beyond their initial diagnosis and introduced the potential for secondary cancers induced by therapy [12]. It is possible that the incidence of treatment-induced cancers may increase in the future. Long-term toxicity of cancer treatments both from systemic chemotherapy and radiation therapy become an important survivorship concern for patients and their physicians.

There is a paucity of data regarding the risk of secondary cancer attributable to modern radiation therapy. In this retrospective cohort study, our primary objective was to assess the risk of breast cancer patients developing an ipsilateral lung cancer and the risk of lung cancer patients developing an ipsilateral breast cancer. We also sought to determine the predominant tumor subtypes and survival outcomes of patients in both clinical scenarios.

\section{Methods}

We conducted a single institution retrospective review of patients treated at the 
Ottawa Hospital Cancer Centre between 1995 and 2013. Patients included in the study were diagnosed with both breast cancer and lung cancer primaries within this period. Subjects were included irrespective of order in which primaries were diagnosed and length of time between diagnoses. Patients with distant metastatic disease were excluded. Ethics approval for the project was obtained through the Ottawa Hospital Research Ethics Board.

The chart review identified a total of 261 patients, of whom 252 were deemed eligible for study inclusion. Six patients were excluded due to secondary metastases and three patients were excluded who had mediastinal masses rather than lung primaries. Data from the medical chart of each patient were extracted. Information was recorded pertaining to patient demographics, cancer location and subtype, tumor stage, surgery, radiotherapy, systemic treatment, dates of diagnoses, lifestyle factors, past medical history, cancer recurrence and patient outcome. The same data was obtained for the subsequent primary.

Data were analyzed to produce basic descriptive information, bivariate and survival outcomes. Fisher's Exact test was used to evaluate correlation between laterality of the second cancer primary and radiation treatment site. Survival data were assessed with Kaplan-Meier analyses.

\section{Results}

All patients (252) were women with most individuals developing a breast primary first (BPF) and fewer diagnosed with a lung primary first (LPF) (Table 1). Median ages at first and second cancer diagnoses were similar between the two groups. The predominant second primary lung cancer subtype in BPF patients was adenocarcinoma (41\%) and in the LPF cohort, the most common second primary breast cancer subtype was invasive ductal carcinoma (69\%) (Table 2, Table 3). Secondary disease was distinguished as a primary rather than metastasis based on pathology. Median follow-up was longer for the BPF group compared to the LPF cohort (82 months $[\mathrm{BPF}]$ vs. 58 months [LPF]) while the median survival was greater for the LPF group (40 months [LPF] vs. 17 months $[\mathrm{BPF}])$.

The median survival of the BPF group was further broken down by stage of the secondary lung primary (Table 4 ). The median survival of lower staged patients (Table 1 and Table 2) was 42 months, and of higher staged patients (Table 3 and Table 4) was 8 months.

Table 1. Patient and follow-up characteristics.

\begin{tabular}{ccc}
\hline & Breast Primary First & Lung Primary First \\
\hline Patient Number & 217 & 35 \\
Median age at first cancer diagnosis & 65.5 (Range $37-92)$ & 67.2 (Range $47-84)$ \\
Median age at second cancer diagnosis & 71.1 (Range $41-97)$ & 71.3 (Range 52 - 88) \\
Median follow-up (months) & 82 (Range $0-238)$ & 58 (Range 2 - 191) \\
\hline
\end{tabular}


Table 2. Primary tumor staging and subtype for patients who developed a breast primary prior to a lung primary (BPF).

\begin{tabular}{|c|c|c|c|c|}
\hline & \multicolumn{2}{|c|}{ Breast Primary } & \multicolumn{2}{|l|}{ Lung Primary } \\
\hline \multirow{5}{*}{ Tumor staging } & Stage 0 & $22(10 \%)$ & Stage I & $62(29 \%)$ \\
\hline & Stage I & $106(49 \%)$ & Stage II & $29(13 \%)$ \\
\hline & Stage II & $75(35 \%)$ & Stage III & $44(20 \%)$ \\
\hline & Stage III & $8(4 \%)$ & Stage IV & $82(38 \%)$ \\
\hline & Stage IV & $6(2 \%)$ & & \\
\hline \multirow{6}{*}{ Tumor subtypes $^{a}$} & IDC & $176(81 \%)$ & Adenocarcinoma & $88(41 \%)$ \\
\hline & ILC & $15(7 \%)$ & NSCLC NOS & $51(24 \%)$ \\
\hline & DCIS & $21(10 \%)$ & Squamous cell carcinoma & $34(15 \%)$ \\
\hline & IDC/ILC (mammary) & $3(1 \%)$ & Small cell lung cancer & $30(14 \%)$ \\
\hline & Spindle Cell & $1(0.5 \%)$ & Large cell lung cancer & $11(5 \%)$ \\
\hline & Phyllodes & $1(0.5 \%)$ & Carcinoid & $3(1 \%)$ \\
\hline
\end{tabular}

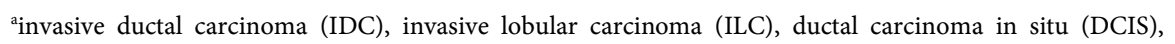
non-small cell lung cancer not otherwise specified (NSCLC NOS).

Table 3. Primary tumor staging and subtype for patients who developed a lung primary prior to a breast primary (LPF).

\begin{tabular}{ccccc}
\hline \multicolumn{2}{c}{ Lung Primary } & \multicolumn{2}{c}{ Breast Primary } \\
\hline \multirow{2}{*}{ Tumor staging } & Stage I & $18(51 \%)$ & Stage 0 & $2(6 \%)$ \\
& Stage II & $6(17 \%)$ & Stage I & $15(43 \%)$ \\
& Stage III & $6(17 \%)$ & Stage II & $13(37 \%)$ \\
Stage IV & $5(14 \%)$ & Stage III & $4(11 \%)$ \\
& & & Stage IV & $1(3 \%)$ \\
Tumor subtypes & & IDC & $24(69 \%)$ \\
& Squamocarcinoma & $19(54 \%)$ & ILC & $7(20 \%)$ \\
& NSCLC NOS & $6(17 \%)$ & DCIS & $2(6 \%)$ \\
& Small cell lung cancer & $2(6 \%)$ & Carcinoma NOS & $2(6 \%)$ \\
& Carcinoma NOS & $1(3 \%)$ & & \\
\hline
\end{tabular}

${ }^{b}$ non-small cell lung cancer not otherwise specified (NSCLC NOS), carcinoma not otherwise specified (carcinoma NOS), invasive ductal carcinoma (IDC), invasive lobular carcinoma (ILC), ductal carcinoma in situ (DCIS)

In the BPF cohort, $65 \%$ of the patients received breast-conserving surgery while $29 \%$ had a mastectomy. The BPF group had a higher proportion of patients who received radiation treatment in comparison to the LPF patients $(67 \%$ [BPF] vs. 51\% [LPF]; Table 4) as well as more patients who were irradiated on the same side as the second cancer primary (52\% [BPF] vs. $39 \%[\mathrm{LPF}])$. The number of patients with a history of smoking was similar in both groups. There 
Table 4. Treatment and survival data.

\begin{tabular}{ccc}
\hline & Breast Primary First & Lung Primary First \\
\hline $\begin{array}{c}\text { Smoking history n } \\
\text { Radiation treatment } \mathbf{n}\end{array}$ & $198(91 \%)$ & $31(89 \%)$ \\
Ipsilateral radiation n & $75(52 \%=75 / 145)$ & $18(51 \%=18 / 35)$ \\
Cancer-related mortality (\%) & 77 & 61 \\
$\begin{array}{c}\text { Median survival from second } \\
\text { diagnosis (months) }\end{array}$ & 17 & 40 \\
$\begin{array}{c}\text { Median survival for stage I and II } \\
\text { from second diagnosis (months) }\end{array}$ & 42 & 40 \\
$\begin{array}{c}\text { Median survival for stage III and IV } \\
\text { from second diagnosis (months) }\end{array}$ & 8 & 42 \\
\hline
\end{tabular}

was no correlation between the location of the second cancer and the side that received treatment based on Fisher's exact test $(\mathrm{P}=0.7382[\mathrm{BPF}], \mathrm{P}=0.1141$ $[\mathrm{LPF}])$. In other words, having localized radiation to the chest for either primary did not appear to influence the emergence of a second primary on the ipsilateral side.

The BPF cohort was broken down into patients who developed the second lung primary on the ipsilateral side and contralateral side in reference to the location of radiation treatment (Table 5, Table 6). Of the BPF group, 111 developed an ipsilateral lung cancer and 106 had a contralateral lung cancer. There was no significant difference in lung cancer stage, tumor subtypes, smoking history, radiotherapy, medical therapy, or surgical intervention. Given the low number of LPF patients and lack of power due to this fact, the analysis was not completed for this cohort of patients.

Kaplan-Meier survival data for both cohorts can be seen in Figure 1 and Figure 2. Most of the patients in this study fell in the BPF group with fewer patients in the LPF group. This is in keeping with the lower percentage of lung cancer patients surviving long enough to develop a subsequent breast primary. Those patients who were disease free from their lung cancer diagnosis and developed subsequent breast cancer had a more favorable prognosis and were likely cured of their lung cancer. Overall, the median survival time from the second primary diagnosis was lower in the BPF cohort which is attributable to the shorter survival times associated with lung cancer compared to breast cancer. As seen in our data, the propensity to diagnose lung cancer at a later stage would also contribute to the lower survival lengths and higher cancer-related mortality in the group with a second lung primary. This can be seen in Table 2 and Table 3, as $58 \%$ of BPF patients developed stage III-IV lung cancer, compared to $31 \%$ of LPF patients who initially had stage III-IV lung cancer. Furthermore, this trend is illustrated in Figure 1, where the BPF survival curve drops off much earlier than the LPF survival curve (Figure 2), before leveling off. LPF patients appear to be passing away at a steadier rate compared to the logarithmic curve of BPF survival. 
Table 5. Patient characteristics of the breast primary first cohort based on location of the second primary lung cancer.

\begin{tabular}{|c|c|c|c|c|}
\hline & \multicolumn{2}{|c|}{ Ipsilateral Lung Cancer } & \multicolumn{2}{|c|}{ Contralateral Lung Cancer } \\
\hline Number & \multicolumn{2}{|l|}{111} & \multicolumn{2}{|l|}{106} \\
\hline Median age at breast diagnosis & \multicolumn{2}{|l|}{64.5} & \multicolumn{2}{|l|}{66.2} \\
\hline Median age at lung diagnosis & \multicolumn{2}{|l|}{70.1} & \multicolumn{2}{|l|}{71.5} \\
\hline Smoking history $\mathbf{n}(\%)$ & \multicolumn{2}{|l|}{$97(87)$} & \multicolumn{2}{|l|}{$100(94)$} \\
\hline Breast radiation (\%) & \multicolumn{2}{|l|}{60} & \multicolumn{2}{|l|}{62} \\
\hline $\begin{array}{c}\text { Breast cancer treatment } \\
\text { modalities }(\%)\end{array}$ & $\begin{array}{c}\text { Surgery } \\
\text { Hormonal Therapy } \\
\text { Chemotherapy }\end{array}$ & $\begin{array}{l}94 \\
64 \\
26\end{array}$ & $\begin{array}{c}\text { Surgery } \\
\text { Hormonal Therapy } \\
\text { Chemotherapy }\end{array}$ & $\begin{array}{l}95 \\
63 \\
29\end{array}$ \\
\hline Cancer-related mortality (\%) & 49 & & 42 & \\
\hline $\begin{array}{l}\text { Median survival from lung } \\
\text { diagnosis (months) }\end{array}$ & 24 & & 18 & \\
\hline
\end{tabular}

Table 6. Second primary lung cancer characteristics of the breast primary first cohort.

\begin{tabular}{ccccc}
\hline & \multicolumn{2}{c}{ Ipsilateral Lung Cancer } & \multicolumn{2}{c}{ Contralateral Lung Cancer } \\
\hline \multirow{2}{*}{$\begin{array}{c}\text { Lung cancer } \\
\text { stage }\end{array}$} & Stage I & $37(33 \%)$ & Stage I & $34(32 \%)$ \\
& Stage II & $12(11 \%)$ & Stage II & $16(15 \%)$ \\
& Stage III & $25(23 \%)$ & Stage III & $16(15 \%)$ \\
Lung cancer & Stage IV & $37(33 \%)$ & Stage IV & $40(38 \%)$ \\
tumor subtypes & Squamous cell carcinoma & $18(16 \%)$ & Small cell carcinoma & $17(16 \%)$ \\
& Adenocarcinoma & $47(42 \%)$ & Adenocarcinoma & $38(36 \%)$ \\
& SSCLall cell carcinoma & $14(13 \%)$ & Squamous cell carcinoma & $16(15 \%)$ \\
& Large cell carcinoma & $2(2 \%)$ & Large cell l carcinoma & $7(7 \%)$ \\
& Carcinoid & $2(2 \%)$ & Carcinoid & $1(1 \%)$ \\
\hline
\end{tabular}

cnon-small cell lung cancer not otherwise specified (NSCLC NOS).

The median survival for the LPF cohort was particularly lower than previously reported numbers (Table IV) [13] [14]. This is likely in part due to the lower sample size, giving a weak representation of the overall population. In addition, there were a large proportion of values censored in this cohort due to patients being lost to follow-up which may have impacted on the survival analysis. Given the lower percentage of cancer-related mortality in this group (61\%), earlier death may have also resulted from non-cancer related causes or even the initial lung primary.

BPF patients who developed an ipsilateral lung cancer had a longer median survival compared to contralateral development (Table 5). This could be postulated to be due to effects of radiation on the ipsilateral lung with overall decrease in pulmonary function and potential chronic pneumonitis in the setting of a new lung primary. 


\section{Breast Primary First Survival}

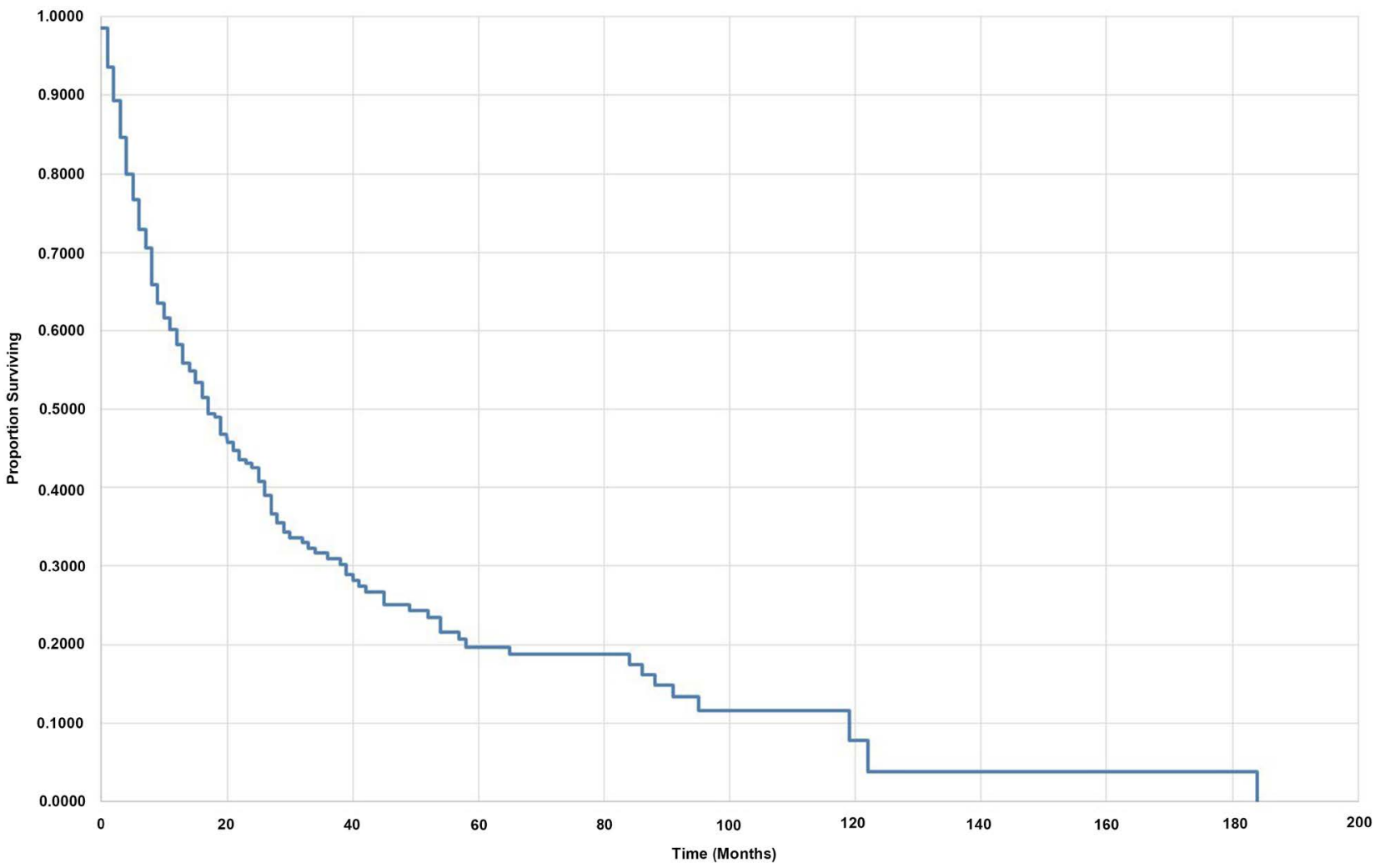

Figure 1. Survival plot for patients who developed a breast primary prior to a lung primary.

\section{Discussion}

\subsection{Survival Data}

Previous clinical trials reported the median survival for lung cancer patients as approximately 120 months for stage I disease, 36 - 60 months for stage II (observation vs. chemo), 15 - 23 months for stage III, and 10 - 12 months for stage IV [13] [14]. In this study's population, BPF patients with stage I and II lung cancer had a median survival of 42 months and those with stage III and IV lung cancer had a median survival of 8 months. The median survival duration in our patient cohort seemed shorter than historical control. The cause for this discrepancy could be several reasons. First of all, the studies referenced looked exclusively at patients who had surgery, while those with poor performance status or who were medically inoperable were excluded. In contrast, the current study took all comers including many unfit patients who could not be treated optimally, or at all. Additionally, the decreased survival time could be in part due to some patients with stage I lung primaries who may also have concurrent metastases from breast cancer. While cancer pathology, tumor site, and time to second diagnoses were taken into consideration to rule out any obvious metastases, lack of data in several patients treated early in the study timeframe made it difficult to completely exclude the presence of metastatic disease. The earlier mortality in these groups could also be attributed to the effect of developing two separate 


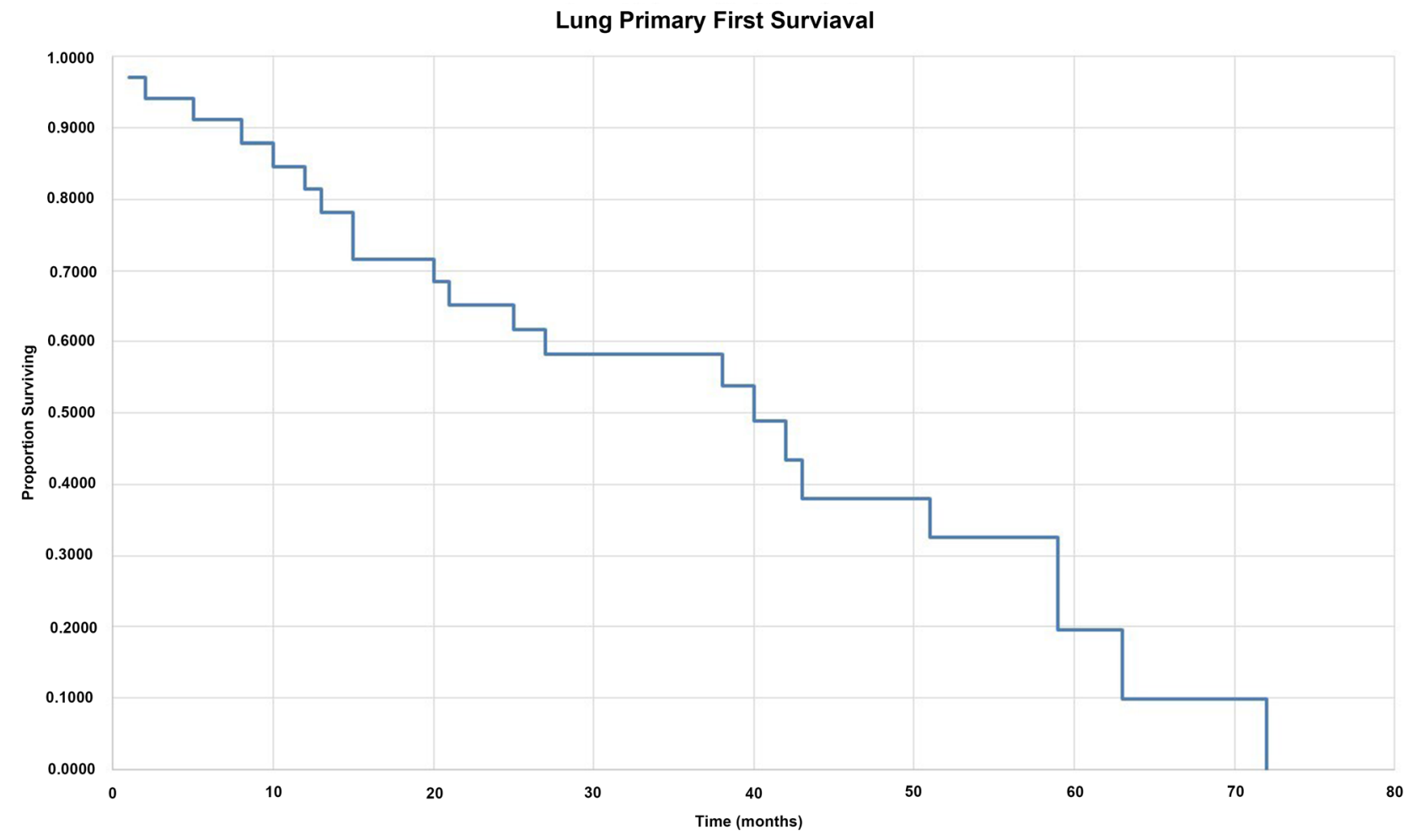

Figure 2. Survival plot for patients who developed a lung primary prior to a breast primary.

cancers and its underlying effect on survival. This is apparent in a study by Schaapveld et al. that found that second primaries in breast cancer patients increased the risk of death, even if the malignancy was considered prognostically favorable [15]. Lastly, this may be attributed to the older patient population, especially when selecting out those who have had sufficient time to develop two primaries. While JBR-10 and RTOG 9309 had median patient ages of 61 and 60 respectively, the current study had a median age of 71 at lung cancer diagnoses.

\subsection{Radiotherapy and Second Primaries}

Early diagnosis and improved radiotherapy treatment options have been vital to lengthening the survival of cancer patients. However, the increased use of radiation has raised concerns regarding second primary development as long-term sequelae of cancer treatment [12].

There have been several population-based studies that support the link between radiotherapy for a primary breast tumor and the risk of a second cancer, either inside or outside the treatment field. In particular, the risk of lung cancer development following breast irradiation has been a topic of interest due the increased incidence of radiation-induced pulmonary malignancies, as well as the anatomical proximity to the breast's treatment field [16]. In an epidemiological study of breast cancer patients from the Thames Cancer Registry, the relative risk (RR) of developing lung cancer was shown to be significantly elevated in the cohort receiving radiotherapy, compared to the group that did not get radiation 
$(\mathrm{RR}=1.62$ at $10-14$ years $)$ [17]. Among patients younger than 50 years old at breast cancer diagnosis, cancer risk was elevated after 10 years with higher standardized incident ratios (SIR) among irradiated compared to non-irradiated patients. This trend was more appreciable at a lower age cohort as there is an increased risk of second primary development in general among younger breast cancer patients following radiotherapy [15].

Conversely, there have been studies showing no substantial risk in developing treatment-related malignancies after breast cancer radiotherapy. In a systematic evaluation of patients in the SEER cancer registries, Berrington de Gonzalez et al. reported that the majority of second cancers in breast cancer survivors were related to genetics and lifestyle factors rather than radiotherapy exposure [18]. In addition, Zablotska et al. suggested that there was no increased risk of lung cancer in women who had radiotherapy after breast-conserving surgery [19].

This study showed no significant association between laterality of radiation treatment and subsequent lung cancer or breast cancer development, as well as no appreciable differences in age, stage, lung tumor subtypes, smoking history, treatment modalities, or cancer-related mortality based on side of irradiation. The findings suggest that while radiotherapy may play a role in development of treatment-related secondary cancer, the effect associated with radiation is overall small compared to other well-established risk factors. The majority of second primaries can be attributed to lifestyle factors such as smoking, gene-environment interactions, genetic mutations, and susceptibility.

\subsection{Advanced Radiation Planning}

Radiation therapy has evolved rapidly over the past two decades with advances in technology that improve treatment conformity while reducing toxicity to surrounding tissues. The use of intensity-modulated radiation therapy (IMRT) has greatly increased in recent years allowing complex dose distributions suitable for avoiding vital structures near the target [12]. Compared to conventional treatment, IMRT decreases high doses to normal tissue due to its improved geometric conformity. However, IMRT delivers a larger number of monitor units and involves more radiation fields with longer irradiation times, increasing extent of normal tissue exposed to low doses [20]. Modalities such as volumetric modulated arc therapy (VMAT) have emerged as quicker methods of delivering IMRT by changing the collimator configuration as the gantry rotates around the patient, but there are still concerns regarding treatment head scatter and total dose. What effect this has on IMRT and its role in second primary cancer incidence is a topic of debate.

This study included patients with cancer diagnoses between 1995 and 2013. IMRT at the Ottawa Hospital Cancer Centre did not come into clinical practice until 2005 with the first few years being strictly trial participants. Due to this later technological emergence and majority of patients being treated for their primary cancer earlier in the timeframe, IMRT would not have a strong influence 
on the patient cohort and their radiotherapy-induced cancer risk within the study period. As patients are continued to be monitored following treatment using these modalities, the threat of more modern therapy-induced malignancies can be better assessed.

\subsection{Limitations of Study}

The current study had several limitations. First, considering the generally low numbers of radiotherapy-induced primaries per patient treated, the sample size was small to analyze this risk. In addition, the patient population was strictly comprised of patients who developed both a primary cancer and a subsequent second primary. This design restricted the level of statistical analyses that could be performed on the data and a matched cohort of patients who did not develop a second malignancy would be needed to further assess the risk factors. Another limitation is the particularly small LPF group. The low incidence of patients who developed a breast primary following an initial lung primary limited the analysis of this data and a study on a larger LPF population would be required to further assess this group.

One important limitation in this study is the length of follow up. Most current data show the latent period for the development of radiotherapy-induced cancer to be over 10 years. However, for a portion of the study patients this threshold was not reached. Many of the patients who were irradiated may have the potential to develop second primaries outside of the study period and would need to be observed over a longer time interval to further characterize the risk of developing therapy-induced cancers.

\section{Conclusions}

In this retrospective analysis, we evaluated the possible association between oncologic treatment, and the development of secondary malignancies. The current study showed no significant association between laterality of radiation treatment and subsequent lung or breast cancer development, as well as no appreciable differences in age, stage, tumor subtypes, smoking history, treatment modality, or cancer-related mortality based on the side of the second lung cancer.

Radiotherapy has become a standard adjuvant option in the treatment of breast and lung cancer, contributing to longer survival times in patients with one or both malignancies. Previous literature shows a small but significant risk in developing second primary cancers many years after radiation administration, but our centre's data failed to confirm this association keeping in mind the nuances of this unique cancer population. Recent advances in radiotherapy modalities show promise in improved imaging and dose conformity, but the overall threat to proximal structures is still unknown at this point. With IMRT playing a prominent role in standard treatment and the patient population reaching latency periods that correspond to the development of radiation-induced malignancies, the risk attributable to newer technologies can be 
better described.

Given all the lifestyle, environmental and genetic factors which play a role in cancer risk, radiotherapy is simply one of the many contributing factors to second primary development. Larger scale observational studies with longer follow up time are required to further assess the risk of radiation-induced malignancies, especially in younger patient populations. With the current data, the benefits of radiation therapy in appropriate selected patients still outweigh the risks and side effects of treatment. While an effort should be taken to reduce the amount of radiation a patient is subjected to, cancer treatment and prevention with appropriate therapy should always take priority with an appreciation of patient risk factors and latency periods.

\section{Acknowledgements}

Funding support provided by the Ottawa Hospital Research Institute (OHRI).

\section{Conflict of Interest Disclosures}

We declare that we have no conflict of interest.

\section{References}

[1] Harvey, E.B. and Brinton, L.A. (1985) Second Cancer Following Cancer of the Breast in Connecticut, 1935-82. NCI Monogr, 68, 99-112.

[2] Ewertz, M. and Mouridsen, H.T. (1985) Second Cancer Following Cancer of the Female Breast in Denmark, 1943-1980. NCI Monogr, 68, 325-329.

[3] Teppo, L., Pukkala, E. and Saxen, E. (1985) Multiple Cancer-An Epidemiological Exercise in Finland. JNCI, 75, 207-217.

[4] Murakami, R., Hiyama, T., Hanai, A. and Fujimoto, I. (1987) Second Primary Cancers Following Female Breast Cancer in Osaka, Japan-A Population Based Cohort Study. Japanese Journal of Clinical Oncology, 17, 293-302.

[5] Brenner, H., Siegle, S., Stegmeier, C. and Ziegler, H. (1993) Second Primary Neoplasms Following Breast Cancer in Saarland, Germany, 1968-1987. European Journal of Cancer, 10, 1410-1414. https://doi.org/10.1016/0959-8049(93)90013-6

[6] Levi, F., Randimbison, L., Te, V.C., Rolland-Portal, I., Franceschi, S., et al. (1993) Multiple Primary Cancers in the Vaud Cancer Registry, Switzerland, 1974-89. British Journal of Cancer, 67, 391-395. https://doi.org/10.1038/bjc.1993.72

[7] Ford, D. and Easton, D.F. (1995) The Genetics of Breast and Ovarian Cancer. British Journal of Cancer, 72, 805-812. https://doi.org/10.1038/bjc.1995.417

[8] Thompson, D.E., Mabuchi, K., Ron, E., Soda, M., Yokunaga, M., et al. (1994) Cancer Incidence in Atomic Bomb Survivors. Part II: Solid Tumors, 1958-1987. Radiation Research, 137, 17-67. https://doi.org/10.2307/3578892

[9] Travis, L.B., Curtis, R.E., Bennet, W.P., Hankey, B.F., Travis, W.D., et al. (1995) Lung Cancer after Hodgkin's Disease. JNCI, 87, 1324-1327. https://doi.org/10.1093/jnci/87.17.1324

[10] Wagoner, J.K., Archer, V.E., Lundin, F.E., Holaday, D.A. and Lloyd, J.W. (1965) Radiation as a Cause of Lung Cancer among Uranium Miners. The New England Journal of Medicine, 273, 181-188. https://doi.org/10.1056/NEJM196507222730402 
[11] Rivina, L. and Schiestl, R. (2013) Mouse Models of Radiation-Induced Cancers. Advances in Genetics, 84, 83-122. https://doi.org/10.1016/B978-0-12-407703-4.00003-7

[12] Lee, B., Lee, S., Sung, J. and Yoon, M. (2014) Radiotherapy-Induced Secondary Cancerrisk for Breast Cancer: 3D Conformal Therapy versus IMRT versus VMAT. Journal of Radiological Protection, 34, 325-331. https://doi.org/10.1088/0952-4746/34/2/325

[13] Butts, C.A., Ding, K., Seymour, L., Twumasi-Ankrah, P., Graham, B., et al. (2010) Randomized Phase III Trial of Vinorelbine plus Cisplatin Compared with Observation in Completed Resected Stage IB and II Non-Small-Cell Lung Cancer: Updated Survival Analysis of JBR-10. Journal of Clinical Oncology, 28, 29-34. https://doi.org/10.1200/JCO.2009.24.0333

[14] Albain, K.S., Swann, R.S., Rusch, V.R., Turrisi, A.T., Shepher, F.A., et al. (2009) Radiotherapy plus Chemotherapy with or without Surgical Resection for Stage III Non-Small Cell Lung Cancer. Lancet, 374, 379-386. https://doi.org/10.1016/S0140-6736(09)60737-6

[15] Schaapveld, M., Visser, O., Louwman, M.J., de Vries, E.G., Willemse, P.H., et al. (2008) Risk of New Primary Nonbreast Cancers after Breast Cancer Treatment: A Dutch Population-Based Study. Journal of Clinical Oncology, 26, 1239-1246. https://doi.org/10.1200/JCO.2007.11.9081

[16] Marcu, L.G., Santos, A. and Bezak, E. (2014) Risk of Second Primary Cancer after Breast Cancer Treatment. European Journal of Cancer Care, 23, 51-64. https://doi.org/10.1111/ecc.12109

[17] Evans, H.S., Lewis, C.M., Robinson, D., Bell, C.J., Moller, H., et al. (2001) Incidence of Multiple Primary Cancers in a Cohort of Women Diagnosed with Breast Cancer in Southeast England. British Journal of Cancer, 84, 435-440.

https://doi.org/10.1054/bjoc.2000.1603

[18] Berrington de Gonzalez, A., Curtis, R.E., Gilbert, E., Berg, C.D., Smith, S.A., et al. (2010) Second Solid Cancers after Radiotherapy for Breast Cancer in SEER Cancer Registries. British Journal of Cancer, 102, 220-226. https://doi.org/10.1038/sj.bjc.6605435

[19] Zablotska, L.B. and Neugut, A.I. (2003) Lung Carcinoma after Radiation Therapy in Women Treated with Lumpectomy or Mastectomy for Primary Breast Carcinoma. Cancer, 97, 1404-1411. https://doi.org/10.1002/cncr.11214

[20] Hall, E.J. and Phil, D. (2006) Intensity-Modulated Radiation Therapy, Protons, and the Risk of Second Cancers. International Journal of Radiation Oncology, Biology, Physics, 65, 1-7. https://doi.org/10.1016/j.ijrobp.2006.01.027 